\title{
Compressed sensing acceleration of cardiac cine imaging allows reliable and reproducible assessment of volumetric and functional parameters of the left and right atrium
}

\author{
Sebastian Altmann ${ }^{1,2} \cdot$ Moritz C. Halfmann ${ }^{1,2} \cdot$ Ibukun Abidoye $^{1,3} \cdot$ Basel Yacoub $^{4} \cdot$ Michaela Schmidt $^{5}$. \\ Philip Wenzel $^{2,6} \cdot$ Christoph Forman $^{5} \cdot$ U. Joseph Schoepf ${ }^{4} \cdot$ Fei Xiong $^{5} \cdot$ Christoph Dueber $^{1} \cdot$ Karl-Friedrich Kreitner $^{1}$. \\ Akos Varga-Szemes ${ }^{4} \cdot$ Tilman Emrich $^{1,2,4}$ (1)
}

Received: 21 August 2020 / Revised: 26 January 2021 / Accepted: 24 February 2021 / Published online: 29 March 2021

(C) The Author(s) 2021

\begin{abstract}
Objectives To compare volumetric and functional parameters of the atria derived from highly accelerated compressed sensing (CS)-based cine sequences in comparison to conventional (Conv) cine imaging.

Methods CS and Conv cine sequences were acquired in 101 subjects ( 82 healthy volunteers (HV) and 19 patients with heart failure with reduced ejection fraction (HFrEF)) using a 3T MR scanner in this single-center study. Time-volume analysis of the left (LA) and right atria (RA) were performed in both sequences to evaluate atrial volumes and function (total, passive, and active emptying fraction). Inter-sequence and inter- and intra-reader agreement were analyzed using correlation, intraclass correlation (ICC), and Bland-Altman analysis.

Results CS-based cine imaging led to a $69 \%$ reduction of acquisition time. There was significant difference in atrial parameters between CS and Conv cine, e.g., LA minimal volume (LAVmin) (Conv $24.0 \mathrm{ml}$ (16.7-32.7), CS $25.7 \mathrm{ml}$ (19.2-35.2), $p<0.0001$ ) or passive emptying fraction (PEF) (Conv 53.9\% (46.7-58.4), CS 49.0\% (42.0-54.1), $p<0.0001$ ). However, there was high correlation between the techniques, yielding good to excellent ICC (0.76-0.99) and small mean of differences in BlandAltman analysis (e.g. LAVmin - 2.0 ml, PEF 3.3\%). Measurements showed high inter- (ICC > 0.958) and intra-rater (ICC > 0.934) agreement for both techniques. CS-based parameters (PEF AUC $=0.965$, LAVmin AUC $=0.864$ ) showed equivalent diagnostic ability compared to Conv cine imaging (PEF AUC $=0.989$, LAVmin AUC $=0.859)$ to differentiate between HV and HFrEF.

Conclusion Atrial volumetric and functional evaluation using CS cine imaging is feasible with relevant reduction of acquisition time, therefore strengthening the role of CS in clinical CMR for atrial imaging.

Key Points

- Reliable assessment of atrial volumes and function based on compressed sensing cine imaging is feasible.

- Compressed sensing reduces scan time and has the potential to overcome obstacles of conventional cine imaging.

- No significant differences for subjective image quality, inter- and intra-rater agreement, and ability to differentiate healthy volunteers and heart failure patients were detected between conventional and compressed sensing cine imaging.
\end{abstract}

Keywords Heart atria $\cdot$ Magnetic resonance imaging $\cdot$ Atrial function $\cdot$ Heart failure

Tilman Emrich

emrich@musc.edu

1 Department of Diagnostic and Interventional Radiology, University Medical Center of the Johannes Gutenberg-University Mainz, Langenbeckstraße 1, 55131 Mainz, Germany

2 German Center for Cardiovascular Research (DZHK), Partner Site Rhine-Main, Mainz, Langenbeckstraße 1, 55131 Mainz, Germany

3 Afe Babalola University/Multisystem Hospital, Km 8.5, Afe Babalola way, Ado-Ekiti, Ekiti, Nigeria
4 Department of Radiology and Radiological Science, Medical University of South Carolina, 25 Courtenay Drive, Charleston, SC 29425, USA

5 Cardiac MR R\&D, Siemens Healthcare GmbH, Henkestraße, 127, 91052 Erlangen, Germany

6 Center for Cardiology, Cardiology I, University Medical Center Mainz, Langenbeckstraße 1, 55131 Mainz, Germany 


$\begin{array}{ll}\text { Abbreviations } \\ \text { AEF } & \text { Active emptying fraction } \\ \text { AUC } & \text { Area under the curve } \\ \text { BMI } & \text { Body mass index } \\ \text { BSA } & \text { Body surface area } \\ \text { bSSFP } & \text { Balanced steady-state free precession } \\ \text { CMR } & \text { Cardiac magnetic resonance imaging } \\ \text { CONV } & \text { Conventional } \\ \text { CS } & \text { Compressed sensing } \\ \text { CTA } & \text { Computed tomography angiography } \\ \text { EDVi } & \text { End-diastolic volume index } \\ \text { EF } & \text { Ejection fraction } \\ \text { ESVi } & \text { End-systolic volume index } \\ \text { HFpEF } & \text { Heart failure with preserved ejection fraction } \\ \text { HFrEF } & \text { Heart failure with reduced ejection fraction } \\ \text { HV } & \text { Healthy volunteers } \\ \text { ICC } & \text { Intraclass correlation } \\ \text { LA } & \text { Left atrium } \\ \text { LAVmax } & \text { Left atrial maximal volume } \\ \text { LAVmin } & \text { Left atrial minimal volume } \\ \text { LoA } & \text { Limits of agreement } \\ \text { MyoMass } & \text { Myocardial mass index } \\ \text { PEF } & \text { Passive emptying fraction } \\ \text { RA } & \text { Right atrium } \\ \text { RAVmax } & \text { Right atrial maximal volume } \\ \text { RAVmin } & \text { Right atrial minimal volume } \\ \text { ROC } & \text { Receiver operating characteristic } \\ \text { SVi } & \text { Stroke volume index } \\ \text { TEF } & \text { Total emptying fraction } \\ & \end{array}$

\section{Introduction}

Left and right ventricular volumes and function are important indicators for the severity of cardiac diseases, and cardiac magnetic resonance imaging (CMR) is considered reference standard for volumetric assessment [1]. Quantification of atrial volumes and function is of increasing importance for diagnostic and prognostic purposes in various cardiac diseases such as heart failure with preserved or reduced ejection fraction (HFpEF and HFrEF), and dilated and hypertrophic cardiomyopathies [2-5]. In addition, atrial volumes can be predictive markers in several cardiomyopathies, and elevated atrial volumes increase the risk for several concomitant cardiac diseases, e.g., atrial fibrillation, and are correlated with an increased failure rate in related interventions [6-8]. Therefore, atrial volumes can be utilized as indicators for risk management [9] and as a guide for therapeutic decisions, for example indication for mitral valve surgery [10]. Beside the estimation of atrial volumes, assessment of more sophisticated parameters such as total, active, and passive emptying fraction have gained scientific and clinical attention, especially in the setting of diagnosis and treatment of atrial fibrillation [11].

Thus, an efficient and accurate way to determine atrial volumes and function is crucial to enable optimal patient management. The calculation of atrial volumes can be performed by echocardiography, computed tomography angiography (CTA), and CMR. CTA carries the risk of radiation and contrast exposure, while echocardiography is highly user dependent and suffers from patient-specific factors such as poor acoustic windows. As of today, CMR is considered the noninvasive reference standard for atrial volumetric assessment [12-15]. The first paper eliciting the assessment of atrial volumes using CMR was published in 1993 [16]. Since then, CMR techniques have been established in clinical routine because of their excellent reproducibility and accuracy [17-20]. Current standard volumetric evaluation is predominantly performed using balanced steady-state free precession (bSSFP)based conventional cine imaging. Conventional cine imaging represents a multi-heartbeat segmented CMR image acquisition approach, i.e., image information acquired over multiple cardiac cycles are fused into a single image. Therefore, conventional cine imaging (a) can be prone to artifacts due to irregular heartbeats and (b) is time-consuming as several heartbeats are needed to gather all required data [21]. Such limitations have recently been addressed by highly accelerated acquisition techniques, such as compressed sensing (CS) by reducing the number of required heartbeats to gain all needed information. CS acceleration is achieved by using random under-sampling of $\mathrm{k}$-space and exploiting the compressibility of medical images in a transform domain during a non-linear iterative reconstruction [22]. With a CS-based cine method, a complete volumetric assessment of the heart is feasible in a single breath-hold. Currently, experience with CS cine is limited to clinical feasibility studies related to left ventricular function and volumes [23-25]. Our hypothesis was that CSaccelerated cine imaging allows accurate atrial imaging. The purpose of this study was to evaluate the agreement in atrial volumes and function between CS-based and conventional cine imaging in HFrEF patients and healthy volunteers (HVs). In addition, we evaluated the impact of CS on interand intra-reader agreement and investigated if there are differences between both cine sequences in the ability to discriminate healthy volunteers from HFrEF patients.

\section{Methods}

\section{Study participants}

The protocol of this single-center study was approved by our institutional ethics committee, and all participants provided written informed consent. A total of 101 subjects were 
Table 1 Comparison of pulse sequence parameters between conventional and CS cine imaging

\begin{tabular}{lcc}
\hline Parameter & Conventional & CS \\
\hline Repetition time (ms) & 37.68 & 39.20 \\
Echo time (ms) & 1.39 & 1.21 \\
Reconstructed cardiac phases & 25 & 25 \\
Field of View (mm) & 360 & 370 \\
Flip angle (deg) & 60 & 34 \\
Voxel size (mm $\left.{ }^{3}\right)$ & $1.5 \times 1.5 \times 8.0$ & $1.5 \times 1.5 \times 8.0$ \\
Cardiac cycle/slice & $1+9^{*}$ & $1+2^{*}$ \\
Acceleration factor & $3(\mathrm{GRAPPA})$ & $6.5(\mathrm{CS})$ \\
\hline
\end{tabular}

*First heartbeat is used for signal preparation, $9 / 2$ heartbeats are used for data acquisition

enrolled including $82 \mathrm{HVs}$ and 19 consecutive patients with non-ischemic HFrEF. HFrEF patients were recruited between August 2018 and January 2019. Subjects with no history of cardiovascular events or symptoms, no cardiovascular risk factors (e.g., hypertension or diabetes), and normal cardiac parameters (normal left and right ventricular volumes, normal myocardial $\mathrm{T} 1$ and $\mathrm{T} 2$ relaxation times according to our institutional reference ranges, absence of late gadolinium enhancement) were considered healthy. No HV was excluded from the study due to abnormal CMR findings. HFrEF patients with a broad range of atrial volumes and function were selected to ensure validation of CS cine images that includes healthy to severely impaired atria.

\section{CMR imaging}

All patients underwent conventional cine and CS cine imaging on a 3T system (MAGNETOM Prisma, Siemens Healthcare). Standard cardiac views (2- and 4-chamber) were recorded using a unified imaging protocol with a conventional accelerated (GeneRalized Autocalibrating Partial Parallel Acquisition, GRAPPA with an acceleration factor of 3) product sequence and a CS-accelerated cine prototype pulse sequence (CS acceleration factor: 6.5), both using retrospective ECG gating during expiratory breath-hold. The total acquisition time including scan time and pauses for recovery and breathing commands for both sequences was recorded. Additional pulse sequence parameters are reported in Table 1.

In addition to the CMR sequences used for atrial assessment, the following acquisitions were performed. A short-axis stack of conventional cine images in expiratory breath-hold was acquired to obtain ventricular volumes and function. T1 mapping (modified LookLocker inversion-recovery with 5(3)3-scheme, TR/TE 280.56/1.12 ms; FOV $360 \mathrm{~mm}$; slice thickness $8 \mathrm{~mm}$; and flip angle $35^{\circ}$ ), T2 mapping (three T2 preparation pulses with a duration of $0.0,30.0$, and $55.0 \mathrm{~ms}$ and a recovery period of 3 heart beats; TR/TE 207.39/
Fig. 1 Image example for atrial segmentation in Conv $(\mathbf{a}+\mathbf{c})$ and $\mathrm{CS}$ cine imaging $(\mathbf{b}+\mathbf{d})$
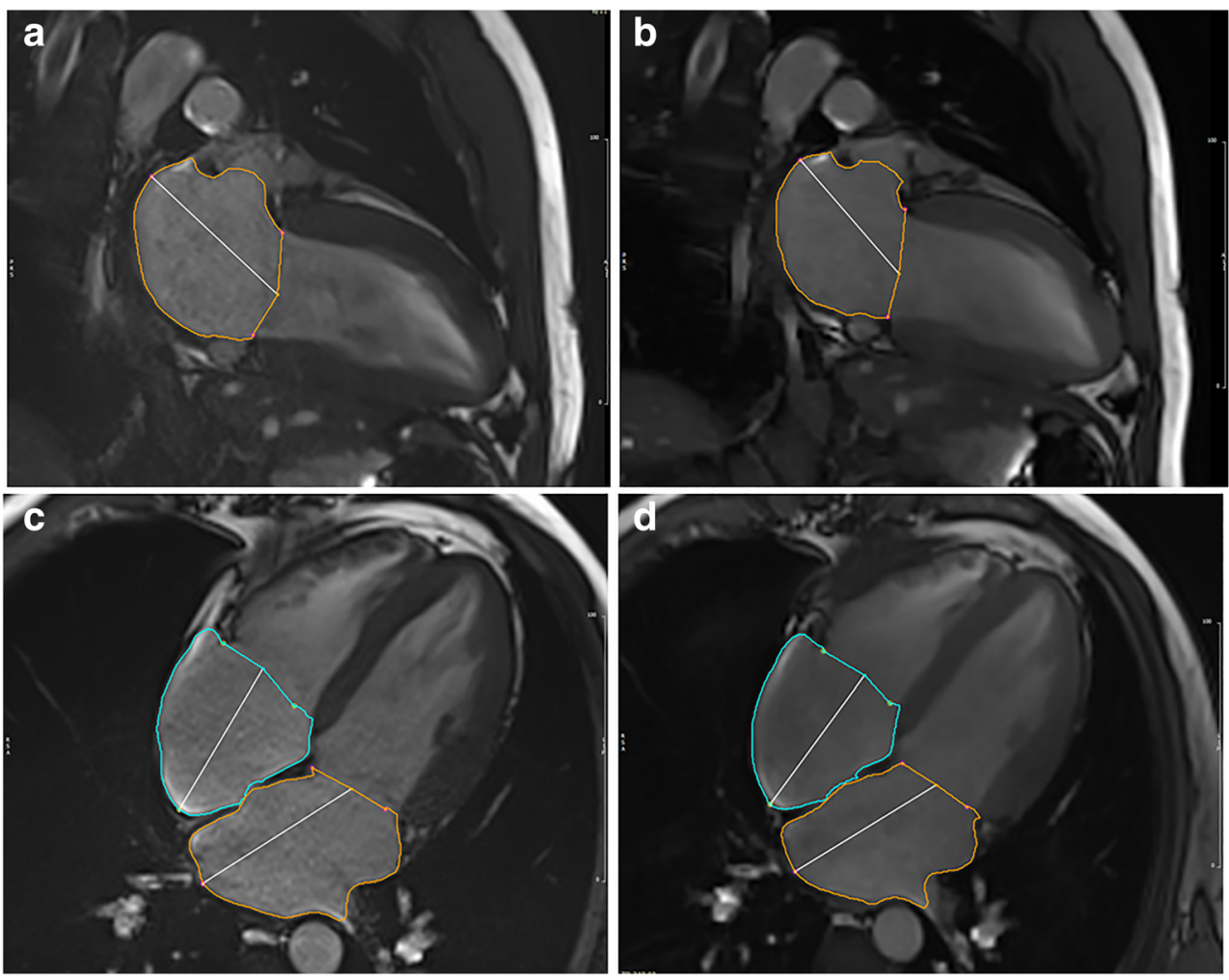
Fig. 2 Time-volume curve for the late atrium indicating functional parameters: Vmax, Vmin, Vmin (mid-diastole), Vmax (middiastole)

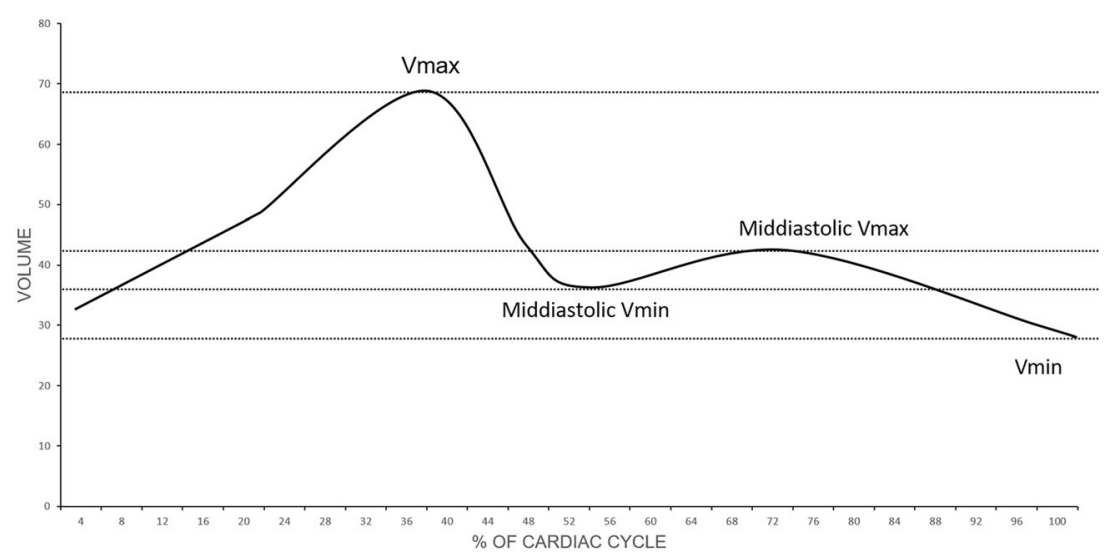

$1.32 \mathrm{~ms}$; FOV $360 \mathrm{~mm}$; slice thickness $8 \mathrm{~mm}$; and flip angle $\left.12^{\circ}\right)$, and post-contrast $(0.2 \mathrm{mmol} / \mathrm{kg}$ gadoteric acid) late gadolinium enhancement images using a phase-sensitive inversion-recovery technique were acquired in long- and short-axis orientations for myocardial characterization.

\section{Image analysis and post-processing}

Image analysis was performed by a radiology resident with 1 year of experience in CMR, including > 100 CMR examinations and SCMR Level 1 certificate. Dedicated cardiovascular software (cvi42, V5.11, Circle) was used for postprocessing of cine and mapping sequences according to SCMR guidelines [1]. For T1 and T2 mapping, global and septal measurements were obtained. For the assessment of atrial volumes and function, a machine learning-assisted measurement of left and right atrial time-volume curves was performed (Fig. 1). Thus, volumetric analysis was semiautomatically performed with minor manual changes when necessary (second-look procedure). Atrial appendage, pulmonary veins, and the inferior and superior vena cava were excluded, according to standard practice [19, 26-28]. Both conventional cine and CS cine images were used to evaluate maximal and minimal left atrial volumes using the biplane arealength calculation technique, which is the method of choice if Simpson's method is not practical [29]. The volume of the right atrium was solely calculated from a 4-chamber view due to the lack of dedicated right-heart 2-chamber views. According to current literature and the increasing clinical interest in atrial functional parameters, total emptying fraction (TEF), passive emptying fraction (PEF), and active emptying fraction (AEF) were also calculated based on the time-volume curves according to Fig. 2 [30] and the following equations:

i. $\mathrm{TEF}=100 \times(\mathrm{Vmax}-\mathrm{Vmin}) / \mathrm{V} \max$,

ii.PEF $=100 \times($ Vmax - Middiastolic Vmin $) /$ Vmax and

iii.AEF $=100 \times($ Middiastolic Vmax - Vmin $) /$ Middiastolic Vmax
From a (patho-)physiological point of view, TEF represents the atrial reservoir, while PEF represents conduit, and AEF the booster function of the atria [31]. In order to rate intra-observer reliability, 20 randomly selected HV were reevaluated by the primary observer after a hiatus of 1 month to minimize recall bias. For evaluation of interobserver reliability, 39 randomly selected subjects $(n=$ $20 \mathrm{HV}$ and $n=19 \mathrm{HFrEF}$ patients) were evaluated by a consultant radiologist with $>10$ years of experience in CMR. Qualitative image quality was subjectively rated by three independent readers (readers 1 and 2: same as above; reader 3: radiology resident with 2-year experience in CMR, SCMR Level 1 certificate) on a 4-point Likert scale: (1) anatomic and functional evaluation not assessable due to severe image artifacts; (2) anatomic and functional evaluation assessable despite relevant image artifacts; (3) good image quality with minor artifact; (4) excellent image quality without artifacts. Image sharpness was evaluated using the methodology proposed by Larson et al [32]. The edge sharpness between myocardium and blood pool was quantified using the first image in each series at end diastole for both CS and conventional SSFP cine with MATLAB (The Mathworks, Inc.). The original image was firstly cropped into a small region of interest focusing on the septum area. Bilinear interpolation was then applied to resample the image into more pixel numbers in the horizontal and vertical dimensions. With a Deriche algorithm (first-order derivative) [33], an edge image was obtained to detect the maximum local intensity change using a threshold value. Subsequently, Hough transform was used to identify the longest straight line describing the myocardium boundaries, and 8 orthogonal lines were plotted across it to get the signal intensity profiles. Based on the method proposed by Sheal et al [34], the sharpness is defined as the mean inverse distance $(1 /$ d) between $0.8 \times($ I_max - I_min $)+$ I_min and $0.2 \times($ I_max - I_min $)+$ I_min) across the profiles. The workflow is summarized in Figure S1. 


\section{Statistical analysis}

Statistical analyses were performed using SPSS Statistics (V25, IBM Corp.). The Kolmogorov-Smirnov test was used to assess normal distribution of the continuous data. Continuous variables were reported as mean \pm standard deviation if normally distributed, and as median/interquartile range in case of non-normal distribution. Categorical variables were displayed as absolute frequencies and proportions. Differences between conventional cine and CS cine parameters and subjective image quality were assessed using the MannWhitney $U$ test. Correlation was evaluated by the coefficient of determination $\left(R^{2}\right)$. Agreement between the cine sequences and inter- and intra-rater agreement were judged by using intraclass correlation (ICC) analysis with two-way mixed effects and focus on absolute agreement with the level of agreement as follows: poor, ICC $<0.5$; moderate, ICC $=0.5-0.75$; good, ICC $=0.76-$ 0.9 ; excellent, ICC $>0.9$ [35]. Further agreement was tested by Bland-Altman analysis (mean difference, upper and lower limits of agreement (LoA)) [36]. Agreement between readers for subjective image quality was analyzed using Fleiss's kappa with the level of agreement as follows: $k<0$ poor; $k=0.01-0.2$ slight; $k=0.21-0.4$ fair; $k=0.41-0.6$ moderate, $K=0.61-0.8$ substantial; $\kappa>0.81$ almost excellent. To evaluate differences in the ability to discriminate between HV and HFrEF patients, sensitivity and specificity were calculated. Receiver operating characteristic (ROC) curve analysis was used to calculate the area under the curve (AUC). The DeLong method was used for pairwise comparison of ROC curves to determine significant differences between
AUCs. Significant difference was considered at $p$ values less than 0.05 on a local level.

\section{Results}

\section{Study population}

The overall study population consisted of 101 patients (median age: 27 years), including 63 men and 38 women. Detailed baseline characteristics of the study population are displayed in Table 2. There were significant differences between the groups with respect to volumetric and functional parameters of the left ventricle ( $p<0.05$ for all). Mean total acquisition time for the 2-and the 4-chamber views was $41 \mathrm{~s}$ using the conventional cine and $13 \mathrm{~s}$ with CS cine imaging, translating into a $69 \%$ time saving using CS cine imaging.

\section{Comparison between conventional and CS cine imaging}

Volumetric and functional assessments of the left and right atria were available in all 101 cases. Overall, there were significant differences for all assessed variables between conventional and CS cine-based evaluation beside right atrial maximal volume (RAVmax) ( $p=0.387$ for RAVmax, otherwise $p<0.0001)$. However, an excellent correlation for maximal and minimal volumes of the left and right atrium $\left(R^{2}>0.90\right)$ and good to excellent correlation for functional parameters TEF and PEF $\left(R^{2}=0.88-0.90\right)$ were observed. AEF showed a moderate correlation between conventional and CS cine imaging $\left(R^{2}=0.55\right)$. These results were confirmed by good to excellent ICC values (e.g., left atrial minimal volume
Table 2 Baseline characteristics of the study population

\begin{tabular}{|c|c|c|c|c|c|}
\hline & & $\begin{array}{l}\text { All } \\
(n=101)\end{array}$ & $\begin{array}{l}\mathrm{HV} \\
(n=82)\end{array}$ & $\begin{array}{l}\text { HFrEF } \\
(n=19)\end{array}$ & $p$ value* \\
\hline \multicolumn{2}{|c|}{ Age (years) } & 27 [24-37] & $27.7[19.2-35.2]$ & $59.0[53.6-64.3]$ & $<0.0001$ \\
\hline Sex, & Male & $63(62.4 \%)$ & $47(57.3 \%)$ & $16(84.2 \%)$ & n.a. \\
\hline$n(\%)$ & Female & $38(37.6 \%)$ & $35(42.7 \%)$ & $3(15.8 \%)$ & n.a. \\
\hline \multicolumn{2}{|c|}{ BMI $\left(\mathrm{kg} / \mathrm{m}^{2}\right)$} & 23.1 [21.4-25.3] & 23.2 [22.6-23.9] & $25.6[23.7-27.5]$ & 0.005 \\
\hline \multicolumn{2}{|c|}{$\operatorname{BSA}\left(\mathrm{m}^{2}\right)$} & 1.9 [1.86-1.95] & 1.9 [1.83-1.93] & 2.0 [1.89-2.11] & 0.088 \\
\hline \multicolumn{2}{|c|}{ LV EDVi $\left(\mathrm{ml} / \mathrm{m}^{2}\right)$} & $87.3[75.4-102.0]$ & $83.0[80.2-85.8]$ & $153.4[135.7-171]$ & $<0.0001$ \\
\hline \multicolumn{2}{|c|}{ LV ESVI (ml/m²) } & $31.7[28.1-45.0]$ & 31.6 [29.9-33.4] & $107.1[90.4-123.8]$ & $<0.0001$ \\
\hline \multicolumn{2}{|c|}{ LV SVI (ml/m²) } & $51.0[41.9-62.1]$ & $53.0[50.4-55.6]$ & $44.9[36.2-53.6]$ & 0.046 \\
\hline \multicolumn{2}{|c|}{ LV EF (\%) } & $59.0[55.5-64.0]$ & $61.6[60.5-62.8]$ & 30.7 [25.5-35.9] & $<0.0001$ \\
\hline \multicolumn{2}{|c|}{ LV MyoMass $\left(\mathrm{g} / \mathrm{m}^{2}\right)$} & $59.5[50.3-67.0]$ & $56.2[54.1-58.2]$ & $74.2[68.9-79.4]$ & $<0.0001$ \\
\hline
\end{tabular}

$H V$ healthy volunteers, $H F r E F$ heart failure with reduced ejection fraction, $B M I$ body mass index, $L V$ left ventricular, $E D V I$ end-diastolic volume index, $E S V I$ end-systolic volume index, $S V I$ stroke volume index, $E F$ ejection fraction, MyoMass myocardial mass index. * indicates comparison between HV and HFrEF groups. Metric data are reported as median and interquartile range 
Table 3 Comparison between conventional and CS-based volumetric and functional atrial assessment

\begin{tabular}{lccccccc}
\hline & Conventional & CS & $p$ value* & $R^{2}$ & Difference & LoA & ICC \\
\hline LAV min (ml) & 24.0 & 25.7 & $<0.0001$ & 0.98 & -2.0 & $-9.6-5.6$ & 0.99 \\
& {$[16.7-32.7]$} & {$[19.2-35.2]$} & & & & & \\
LAV max (ml) & 70.3 & 69.4 & $<0.0001$ & 0.97 & 3.0 & $-7.8-13.8$ & 0.99 \\
& {$[58.7-88.5]$} & {$[56.5-87.5]$} & & & & & \\
RAV min (ml) & 35.2 & 39.3 & $<0.0001$ & 0.90 & -3.0 & $-16.4-10.4$ & 0.97 \\
& {$[26.2-49.0]$} & {$[27.9-50.3]$} & & & & & \\
RAV max (ml) & 77.6 & 75.9 & 0.387 & 0.90 & -0.8 & $-19.5-17.9$ & 0.97 \\
& {$[63.9-98.8]$} & {$[62.8-99.1]$} & & & & & \\
TEF (\%) & 67.8 & 63.11 & $<0.0001$ & 0.90 & 4.2 & $-5.0-13.4$ & 0.95 \\
& {$[61.6-72.3]$} & {$[55.7-66.7]$} & & & & & \\
PEF (\%) & 53.9 & 49.0 & $<0.0001$ & 0.88 & 3.3 & $-6.8-13.4$ & 0.95 \\
AEF (\%) & {$[46.7-58.4]$} & {$[42.0-54.1]$} & & & & & \\
& 40.3 & 34.4 & $<0.0001$ & 0.55 & 6.99 & $-8.3-22.2$ & 0.76 \\
& {$[35.1-47.0]$} & {$[26.5-38.6]$} & & & & & \\
\hline
\end{tabular}

Conv conventional cine imaging, $C S$ compressed sensing cine imaging, $L O A$ limits of agreement, $I C C$ intraclass correlation coefficient, $L A V$ left atrial volume, $R A V$ right atrial volume, Min minimal, Max maximal, $T E F$ total emptying fraction, $P E F$ passive emptying fraction, $A E F$ active emptying fraction; * indicates comparison between Conv and CS technique. Metric data is reported as median and interquartile range
(LAVmin): ICC $=0.99$ ) and small mean differences and upper and lower limits of agreement (e.g., LAVmin: mean difference $-2.0 \mathrm{ml}$, limits of agreement -9.6 to $5.6 \mathrm{ml}$ ). A detailed overview, scatterplots, and Bland-Altman-plots of representative parameters are presented in Table 3 and Figs. 3, 4, and 5.

\section{Inter- and intra-observer variability and image quality}

Excellent agreement was observed in left (conventional ICC > 0.989 and CS ICC > 0.994) and right atrial (conventional ICC $>0.978$ and CS ICC >0.958) volumetric parameters between the readers. Similarly, strong ICCs were found for the same parameters in the intra-observer analysis. Further details are provided in Table 4. Subjective image quality was comparable between both cine techniques (median rating: conventional 4.0 (3.0-4.0) vs CS 3.33 (3.0-4.0), $p=0.159$ ), with a slightly higher but not statistical different rating for $\mathrm{HV}$ in comparison to $\mathrm{HFrEF}$ patients (conventional HV vs HFrEF: 4.0 (3.0-4.0) vs 3.33 (3.0-4.0), $p=0.271$; CS HV vs HFrEF: 3.67 (3.0-4.0) vs 3.0 (3.0-4.0), $p=0.205)$. Inter-reader agreement for subjective image quality was almost excellent for both techniques (conventional
Fig. 3 Correlation between CSand Conv-based evaluation of volumetric atrial parameters, a left atrial minimal volume; $\mathbf{b}$ left atrial maximal volume; $\mathbf{c}$ right atrial minimal volume; $\mathbf{d}$ right atrial maximal volume
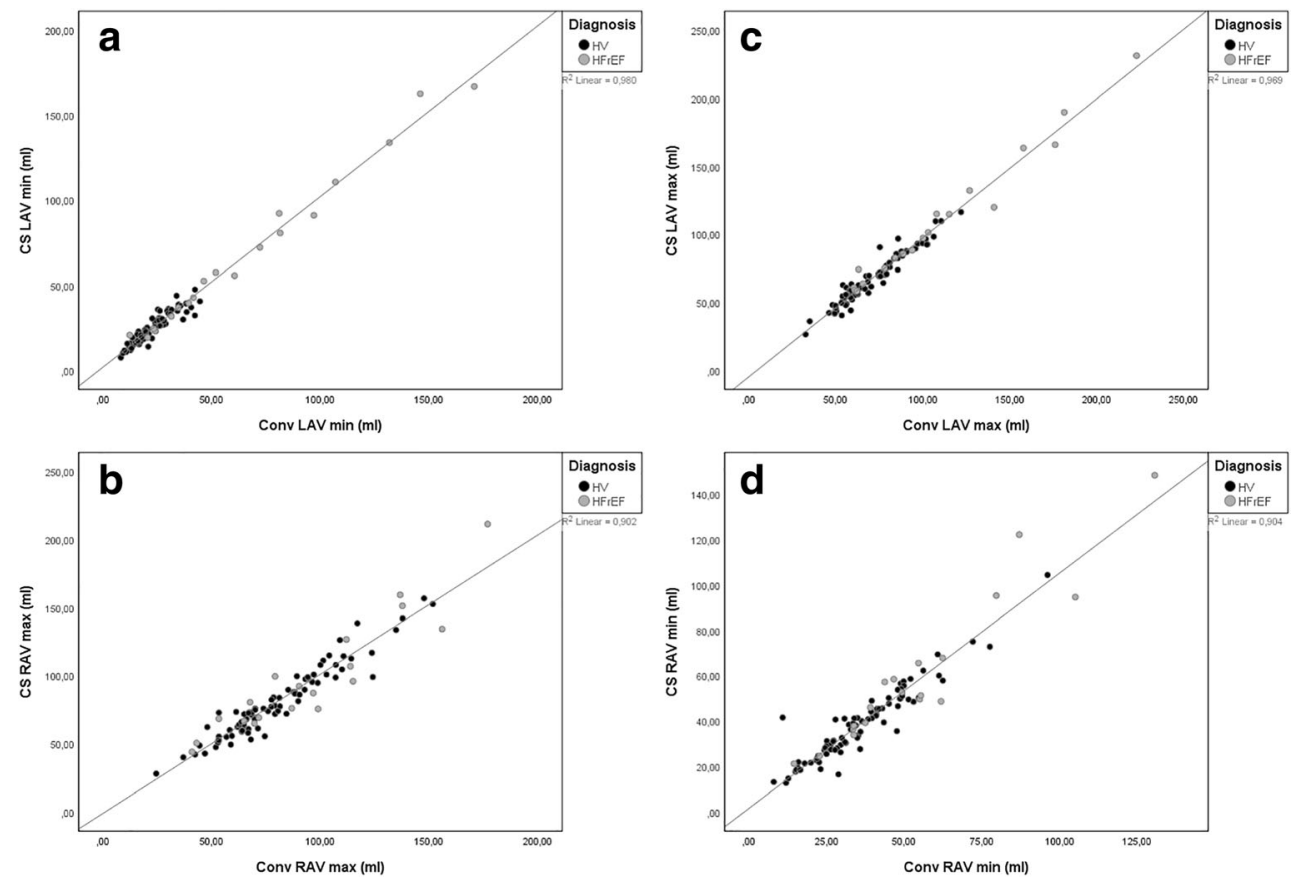

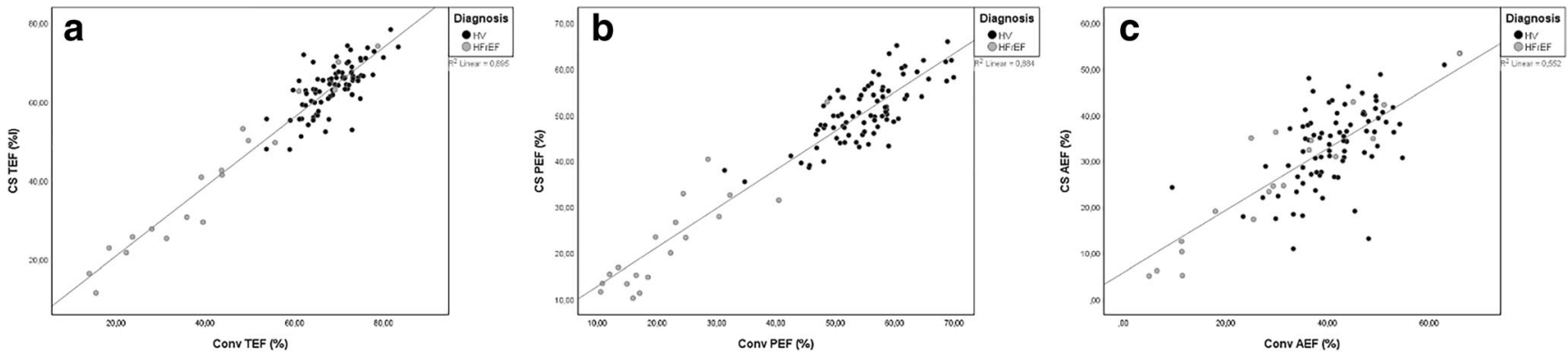

Fig. 4 Correlation between CS- and Conv-based evaluation of functional atrial parameters, a total emptying volume (TEV); b passive emptying volume (PEV); $\mathbf{c}$ active emptying volume (AEV)

$\kappa=0.825, \mathrm{CS} \kappa=0.831)$. Image sharpness was higher for the conventional cine sequence $(0.045 \pm 0.009$ vs. $0.034 \pm$ $\left.0.003 \mathrm{~mm}^{-1}, p=0.005\right)$. An overview of image artifacts is provided in Figure S2 in the supplementary data.

\section{Differentiation between HV and HFrEF}

ROC analysis showed no significant differences between the ability of conventional and CS cine to differentiate HV and HFrEF patients for all parameters. PEF and LAVmin had the highest diagnostic power to differentiate HV from HFrEF patients (PEF: AUC (conventional) $=0.989$ vs AUC (CS) $=0.965 ; p=0.288 ;$ LAVmin: AUC $($ conventional $)=0.859 ;$ AUC $(\mathrm{CS})=0.864 ; p=0.833$ ) (Fig. 6, Table 5).

\section{Discussion}

In this study, we investigated the agreement in atrial volumes and function between conventional cine and novel, highly accelerated CS-based cine imaging in HV and HFrEF patients. We found that the assessment of CS-based atrial parameters is comparable to conventional cine imaging with a high level of agreement between the techniques. Atrial evaluation using CS-based cine images showed excellent inter- and intrareader agreement in comparison with conventional cine imaging-based assessment. There were no significant differences in the ability to differentiate HV and HFrEF patients between the techniques.

As atrial imaging is getting more and more clinically important, the implementation of advanced cardiac imaging for assessment of atrial anatomy and function is intriguing [37].
Fig. 5 Bland-Altman analysis for minimal left and right atrial volumes and total and passive emptying fraction in comparison between Conv and CS cine
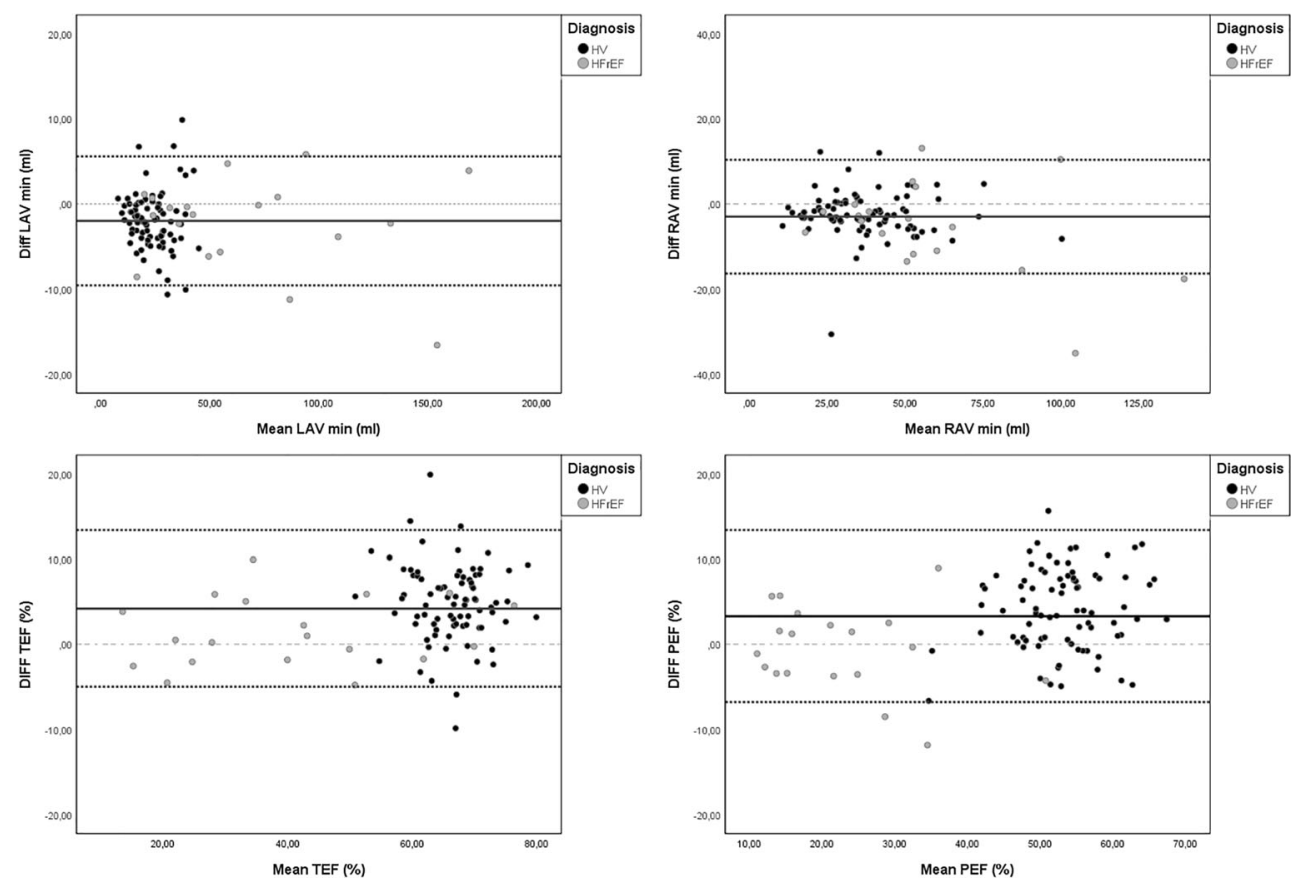
Table 4 Intraclass correlation for inter-and intra-observer variability

\begin{tabular}{llllll}
\hline & \multicolumn{2}{l}{ Inter-observer } & & & \multicolumn{2}{c}{ Intra-observer } \\
\cline { 2 - 3 } & Conventional & CS & & Conventional & CS \\
\hline LAV min & 0.995 & 0.997 & & 0.934 & 0.939 \\
LAV max & 0.989 & 0.994 & & 0.952 & 0.941 \\
RAV min & 0.978 & 0.958 & & 0.969 & 0.976 \\
RAV max & 0.984 & 0.965 & & 0.954 & 0.977 \\
\hline
\end{tabular}

$I C C$ intraclass correlation coefficient, $L A V$ left atrial volume, $R A V$ right atrial volume, Min minimal, Max maximal, Inter inter-observer, Intra intra-observer

Clinically, echocardiography is the most commonly used modality to evaluate atrial size and function. However, CMR is considered gold standard for the volumetric and functional assessment of atria and ventricles because of its high accuracy and reproducibility [12-15]. In view of the growing clinical importance of the volumetric atrial assessment, it becomes pivotal to improve the access to such relevant data. Although CS cine imaging is a relatively new clinical CMR tool, it has already been validated for left ventricular volumetry with convincing results. It has been shown that volumetric assessment, estimation of valve regurgitation, and wall-motion disorders are feasible [24, 25, 38, 39]. Therefore, CS-based cine imaging is a promising method for acceleration of cardiac image acquisitions and holds the potential to be rapidly transferred to routine clinical applications. Our work complements the current knowledge related to CS cine imaging of the ventricles by demonstrating accurate visualization of the fine atrial borders using this highly accelerated technique.

The basic principle of CS is built on random k-space undersampling, a method that creates noise-like artifacts. These
Table 5 Comparison of diagnostic accuracy between conventional and CS-based atrial parameters

\begin{tabular}{llllll}
\hline & Parameter & AUC & Sensitivity & Specificity & $p$ value* \\
\hline LAV min & Conventional & 0.859 & 79.9 & 85.4 & 0.833 \\
& CS & 0.864 & 73.7 & 91.5 & \\
LAV max & Conventional & 0.786 & 63.2 & 84.1 & 0.152 \\
& CS & 0.809 & 78.9 & 69.3 & \\
RAV min & Conventional & 0.732 & 89.5 & 50 & 0.792 \\
& CS & 0.737 & 68.4 & 73.2 & \\
RAV max & Conventional & 0.625 & 57.9 & 63.4 & 0.903 \\
& CS & 0.621 & 89.5 & 32.9 & \\
TEF & Conventional & 0.889 & 93.9 & 84.2 & 0.198 \\
& CS & 0.860 & 93.9 & 78.9 & \\
PEF & Conventional & 0.989 & 97.6 & 94.7 & 0.288 \\
& CS & 0.965 & 92.7 & 94.7 & \\
AEF & Conventional & 0.746 & 92.7 & 63.2 & 0.145 \\
& CS & 0.675 & 84.1 & 52.6 & \\
\hline
\end{tabular}

$A U C$ area under the curve, $L A V$ left atrial volume, $R A V$ right atrial volume, Min minimal, Max maximal, TEF total emptying fraction, $P E F$ passive emptying fraction, $A E F$ active emptying fraction. * DeLong test comparing conventional and CS AUCs

artifacts are successfully reduced utilizing wavelet regularization during the non-linear iterative reconstruction [22]. CS acceleration has been implemented and found feasible in multiple other CMR techniques, such as 4D flow, perfusion imaging, and T1 mapping [40-42]. Other strategies have been described to accelerate cine imaging using k-t acceleration in combination with parallel imaging such as k-t SENSE or k-t GRAPPA [43, 44]. Further developments such as XDGRASP include combination of continuous radial k-space sampling using a golden-angle sampling scheme and multidimensional reconstructions with a CS approach [45]. Notably, clinical validation studies with larger cohorts are
Fig. 6 ROC analysis to differentiate HV and HFrEF comparing the diagnostic accuracy of CS- and Conv-based parameters

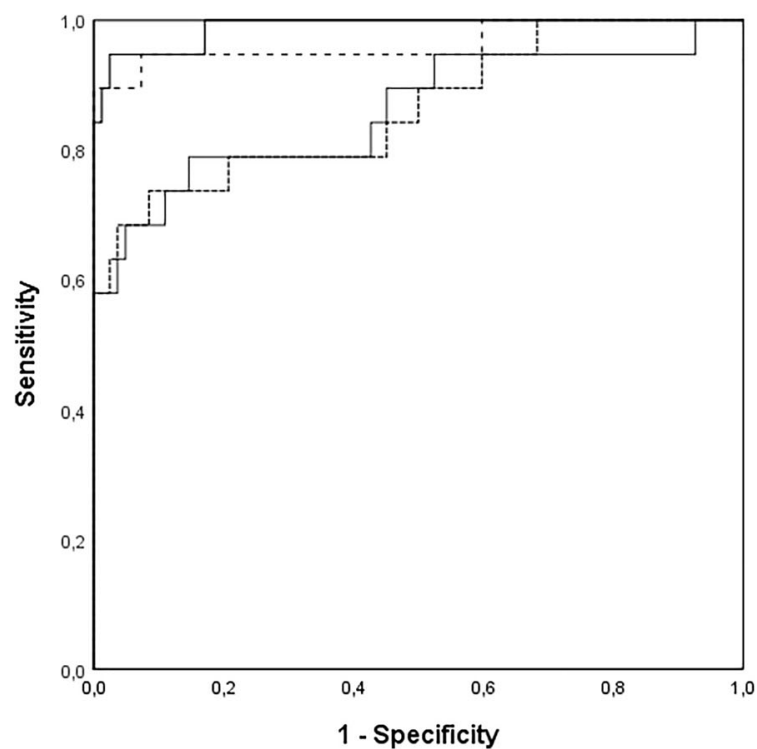

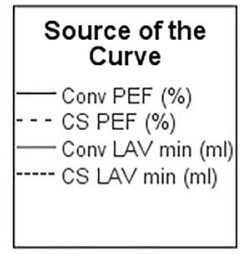


predominantly using CS [25], and no validation data on the performance of atrial imaging for different acceleration strategies have been reported yet.

With the use of CS acceleration, we were able to demonstrate a reduction of mean acquisition time from 41 to $13 \mathrm{~s}$ $(-69 \%)$ in this study for long-axis imaging of the heart. An even higher reduction rate can be achieved when applying CS to short-axis acquisitions, as short-axis coverage of the ventricles needs more slices than long-axis, generally reducing imaging time by $>90 \%$ [25]. Overall, the application of CS cine imaging could especially be beneficial in elderly and diseased patients, who frequently suffer from shortness of breath or arrhythmia. Standard cine imaging, being a summation image of progressively filled $\mathrm{k}$-space data from multiple heartbeats, is susceptible for mistriggering and breathing artifacts. CS cine imaging requires fewer heartbeats for imaging and hence has lower risk for such artifacts, while maintaining the similar spatio-temporal resolution [25]. Therefore, feasibility of CS cine imaging contributes to the process of implementing CS in CMR as a routine clinical tool that decreases acquisition time and reduces the rate of non-diagnostic scans by overcoming the aforementioned limitations of conventional cine imaging. Alternatively, CS could be used to gain more information in a certain amount of time, e.g., increase temporal resolution of functional cardiac imaging. This could be advantageous for more detailed temporal analysis of volume and velocity changes of the heart and more sophisticated evaluations of cardiac function such as deformation imaging or analysis of tissue velocities [46, 47].

In our study, subjective and objective image quality was slightly higher for the conventional technique in comparison to CS-accelerated cine imaging. This can be explained by the combination of several factors, including lower CNR in CS cine due to the lower flip angle and reduced image sharpness. In addition, the majority of our cohort included healthy volunteers with regular sinus rhythm and without breathing artifacts. The number of patients with reduced breath-hold capabilities and/or arrhythmias, who would profit most from CSaccelerated cine imaging, was limited in our study cohort.

The overall correlation and reproducibility of atrial CS imaging in our study was good to excellent with comparable effects to published data from LV and RV analysis [25]. Despite demonstrating excellent inter- and intra-rater reproducibility, minor manual edits of the ML-based atrial segmentations were needed for the vast majority of cases. Subgroup analysis demonstrated that results tend to be better for the HV cohort compared to the HFrEF patients. This might be due to the fact that the machine learning-based assessment of functional parameters performed better in HV because of less motion artifacts, fewer breathing difficulties, and a more typical anatomical appearance of the atria. The HFrEF cohort presented with a subjectively inferior, but still diagnostic image quality and more artifacts, thus more manual corrections had to be performed in order to manage accurate delineation of the atrial contours.

Despite the significant differences in atrial volumes between CS and conventional cine imaging, the disparity was not clinically relevant. The mean difference of the maximum biplane measurements was $3.0 \mathrm{ml}$, whereas the difference of the minimum biplane measurements was only $2.0 \mathrm{ml}$. These differences can be attributed to the combination of technical aspects such as lower CNR and decreased image sharpness of CS-accelerated cine imaging. However, absolute differences between the two cine techniques were comparable to differences between human readers of the same sequences. Clinically, these differences are not likely to affect clinical management of patients. In general, volumetric analyses were more reliable than functional analysis, which incorporates volumes and small volume fractions from different time points of the cardiac cycle. AEF showed the highest deviation and lowest correlation between conventional and CS cine imaging. This may be explained by the nature of this parameter, as small errors in volumetric assessments have a higher impact on parameters that rely on the detailed acquisition of small volumes (differences of around $5 \mathrm{ml}$ ). In addition, our findings corroborate previous studies which have identified AEF as the parameter with the least diagnostic value in comparison to the other functional parameters [48, 49]. Finally, we found no significant differences in differentiating HFrEF patients from HV using CS-based vs. conventional cine-based atrial parameters. In concordance with current literature, minimal volumes and assessment of atrial conduit function by PEF showed the highest diagnostic power to discriminate between healthy and diseased $[49,50]$. Therefore, our results support the clinical implementation of CS cine as a routine clinical tool for atrial imaging.

\section{Limitations}

This study has several limitations. Despite the uneven distribution of healthy volunteers and HFrEF patients in this study, we were still able to demonstrate the applicability of CS cine imaging over a wide range of atrial volumes ranging from 32.6 to $222.8 \mathrm{ml}$. We have chosen a cohort of patients with advanced heart failure as our reference disease cohort and used time-volume analysis for the functional assessment of the atria. Another cohort with less advanced disease and modest effect on atrial size and function, and a more sophisticated functional evaluation, such as atrial strain [51], could be more susceptible to the small measurement errors of CS cine imaging. Therefore, future studies should evaluate $\mathrm{CS}$ cine imaging in other disease cohorts such as HFpEF, including more advanced functional parameters of the atria. Although Simpson's method is considered the gold standard, we used the biplane area-length method in order to calculate the left 
atrial volumes and the single-plane 4-chamber view to calculate the right atrial volume, as it is more frequently used in clinical routine because of faster post-processing time. In addition, the biplane method correlates well with Simpson's method and it is indicated as the method of choice if Simpson's method cannot be performed [29, 52].

\section{Conclusion}

Volumetric and functional atrial evaluation using CS cine imaging is feasible and can be integrated in clinical routine. The implementation of CS cine imaging in clinical routine will allow relevant reduction of CMR examination times, increase productivity of imaging centers while maintaining the assessment of relevant (patho-)physiologic information, therefore strengthening the role of CMR imaging as a reliable diagnostic tool.

Supplementary Information The online version contains supplementary material available at https://doi.org/10.1007/s00330-021-07830-z.

Acknowledgements The authors want to thank Petra Rieck, Kristin Berg, and Lydia Vehling for their technical support of our study.

Funding Open Access funding enabled and organized by Projekt DEAL. PW is supported by a grant from the German Federal Ministry for Education and Research (BMBF 01EO1503). PW is a Principal Investigator of the German Center of Cardiovascular Research (DZHK). Otherwise, the authors state that this work has not received any specific funding.

\section{Declarations}

Guarantor The scientific guarantor of this publication is Tilman Emrich.

Conflict of interest The authors of this manuscript declare relationships with the following companies: MS, CF, and FX are employees of Siemens Healthineers. UJS has received institutional research support and/or honoraria for speaking and consulting from Astellas, Bayer, Bracco, Elucid BioImaging, General Electric, Guerbet, HeartFlow Inc., and Siemens Healthineers. AVS receives institutional research support and travel support from Siemens Healthineers and is consultant for Bayer and Elucid Bioimaging. TE has received a speaker fee and travel support from Siemens Healthineers. All other authors declare no conflict of interest.

Statistics and biometry No complex statistical methods were necessary for this paper.

Informed consent Written informed consent was obtained from all subjects in this study.

Ethical approval Institutional Review Board approval was obtained.
Methodology

- Prospective

- Diagnostic or prognostic study

- performed at one institution

Open Access This article is licensed under a Creative Commons Attribution 4.0 International License, which permits use, sharing, adaptation, distribution and reproduction in any medium or format, as long as you give appropriate credit to the original author(s) and the source, provide a link to the Creative Commons licence, and indicate if changes were made. The images or other third party material in this article are included in the article's Creative Commons licence, unless indicated otherwise in a credit line to the material. If material is not included in the article's Creative Commons licence and your intended use is not permitted by statutory regulation or exceeds the permitted use, you will need to obtain permission directly from the copyright holder. To view a copy of this licence, visit http://creativecommons.org/licenses/by/4.0/.

\section{References}

1. Schulz-Menger J, Bluemke DA, Bremerich J et al (2020) Standardized image interpretation and post-processing in cardiovascular magnetic resonance - 2020 update. J Cardiovasc Magn Reson 22:19

2. Pieske B, Tschöpe C, de Boer RA et al (2019) How to diagnose heart failure with preserved ejection fraction: the HFA-PEFF diagnostic algorithm: a consensus recommendation from the Heart Failure Association (HFA) of the European Society of Cardiology (ESC). Eur Heart J 40:3297-3317

3. Gulati A, Ismail TF, Jabbour A et al (2013) Clinical utility and prognostic value of left atrial volume assessment by cardiovascular magnetic resonance in non-ischaemic dilated cardiomyopathy. Eur J Heart Fail 15:660-670

4. Maron BJ, Haas TS, Maron MS et al (2014) Left atrial remodeling in hypertrophic cardiomyopathy and susceptibility markers for atrial fibrillation identified by cardiovascular magnetic resonance. Am J Cardiol 113:1394-1400

5. Di Tullio MR, Qian M, Thompson JLP et al (2018) Left atrial volume and cardiovascular outcomes in systolic heart failure: effect of antithrombotic treatment. ESC Heart Fail 5:800-808

6. Van Gelder IC, Crijns HJ, Van Gilst WH, Verwer R, Lie KI (1991) Prediction of uneventful cardioversion and maintenance of sinus rhythm from direct-current electrical cardioversion of chronic atrial fibrillation and flutter. Am J Cardiol 68:41-46

7. Benjamin EJ, D'Agostino RB, Belanger AJ et al (1995) Left atrial size and the risk of stroke and death. The Framingham Heart Study. Circulation 92:835-841

8. Helms AS, West JJ, Patel A et al (2009) Relation of left atrial volume from three-dimensional computed tomography to atrial fibrillation recurrence following ablation. Am J Cardiol 103:989-993

9. Tiwari S, Løchen M-L, Jacobsen BK et al (2016) CHA2DS2-VASc score, left atrial size and atrial fibrillation as stroke risk factors in the Tromsø Study. Open Heart 3:e000439

10. Baumgartner H, Falk V, Bax JJ et al (2017) 2017 ESC/EACTS Guidelines for the Management of Valvular Heart Disease. Rev Esp Cardiol (Engl Ed) 71:110

11. Javier OF, Litten B, Chantal de KM et al (2016) Multimodality cardiac imaging for the assessment of left atrial function and the association with atrial arrhythmias. Circ Cardiovasc Imaging 9: e004947

12. Tops LF, Schalij MJ, Bax JJ (2010) Imaging and atrial fibrillation: the role of multimodality imaging in patient evaluation and management of atrial fibrillation. Eur Heart J 31:542-551 
13. Agner BFR, Kühl JT, Linde JJ et al (2014) Assessment of left atrial volume and function in patients with permanent atrial fibrillation: comparison of cardiac magnetic resonance imaging, 320-slice multi-detector computed tomography, and transthoracic echocardiography. Eur Heart J Cardiovasc Imaging 15:532-540

14. Kuchynka P, Podzimkova J, Masek M et al (2015) The role of magnetic resonance imaging and cardiac computed tomography in the assessment of left atrial anatomy, size, and function. Biomed Res Int 2015:247865

15. Ponikowski P, Voors AA, Anker SD et al (2016) 2016 ESC Guidelines for the diagnosis and treatment of acute and chronic heart failure: the Task Force for the diagnosis and treatment of acute and chronic heart failure of the European Society of Cardiology (ESC)Developed with the special contribution of. Eur Heart J 37: 2129-2200

16. Matsuoka H, Hamada M, Honda T et al (1993) Measurement of cardiac chamber volumes by cine magnetic resonance imaging. Angiology 44:321-327

17. Grothues F, Smith GC, Moon JCC et al (2002) Comparison of interstudy reproducibility of cardiovascular magnetic resonance with two-dimensional echocardiography in normal subjects and in patients with heart failure or left ventricular hypertrophy. Am J Cardiol 90:29-34

18. Maceira AM, Prasad SK, Khan M, Pennell DJ (2006) Normalized left ventricular systolic and diastolic function by steady state free precession cardiovascular magnetic resonance. J Cardiovasc Magn Reson 8:417-426

19. Maceira AM, Cosín-Sales J, Roughton M, Prasad SK, Pennell DJ (2013) Reference right atrial dimensions and volume estimation by steady state free precession cardiovascular magnetic resonance. J Cardiovasc Magn Reson 15:29

20. Surkova E, Muraru D, Iliceto S, Badano LP (2016) The use of multimodality cardiovascular imaging to assess right ventricular size and function. Int J Cardiol 214:54-69

21. Axel L, Otazo R (2016) Accelerated MRI for the assessment of cardiac function. Br J Radiol 89:20150655

22. Lustig M, Donoho D, Pauly JM (2007) Sparse MRI: the application of compressed sensing for rapid MR imaging. Magn Reson Med 58:1182-1195

23. Goebel J, Nensa F, Schemuth HP et al (2016) Compressed sensing cine imaging with high spatial or high temporal resolution for analysis of left ventricular function. J Magn Reson Imaging 44:366-374

24. Lin ACW, Strugnell W, Riley R et al (2017) Higher resolution cine imaging with compressed sensing for accelerated clinical left ventricular evaluation. J Magn Reson Imaging 45:1693-1699

25. Vermersch $\mathrm{M}$, Longère $\mathrm{B}$, Coisne $\mathrm{A}$ et al (2020) Compressed sensing real-time cine imaging for assessment of ventricular function, volumes and mass in clinical practice. Eur Radiol 30:609-619

26. Maceira AM, Cosín-Sales J, Roughton M, Prasad SK, Pennell DJ (2010) Reference left atrial dimensions and volumes by steady state free precession cardiovascular magnetic resonance. J Cardiovasc Magn Reson 12:65

27. Le Ven F, Bibeau K, De Larochellière É et al (2016) Cardiac morphology and function reference values derived from a large subset of healthy young Caucasian adults by magnetic resonance imaging. Eur Heart J Cardiovasc Imaging 17:981-990

28. O'Neill J, Swoboda PP, Plein S, Tayebjee MH (2018) Left atrial size and function in a South Asian population and their potential influence on the risk of atrial fibrillation. Clin Cardiol 41:13791385

29. Nacif MS, Barranhas AD, Türkbey E et al (2013) Left atrial volume quantification using cardiac MRI in atrial fibrillation: comparison of the Simpson's method with biplane area-length, ellipse, and three-dimensional methods. Diagn Interv Radiol 19:213-220

30. Maceira AM, Cosin-Sales J, Prasad SK, Pennell DJ (2016) Characterization of left and right atrial function in healthy volunteers by cardiovascular magnetic resonance. J Cardiovasc Magn Reson 18:1-16

31. Blume GG, Mcleod CJ, Barnes ME et al (2011) Left atrial function: physiology, assessment, and clinical implications. Eur J Echocardiogr 12:421-430

32. Larson AC, Kellman P, Arai A et al (2005) Preliminary investigation of respiratory self-gating for free-breathing segmented cine MRI. Magn Reson Med 53:159-168

33. Etienne A, Botnar RM, Van Muiswinkel AMC, Peter Boesiger, Manning WJ, Stuber M (2002) "Soap-Bubble" visualization and quantitative analysis of 3D coronary magnetic resonance angiograms. Magn Reson Med 48:658-666

34. Shea SM, Kroeker RM, Deshpande V et al (2001) Coronary artery imaging: 3D segmented $\mathrm{k}$-space data acquisition with multiple breath-holds and real-time slab following. J Magn Reson Imaging 13:301-307

35. Koo TK, Li MY (2016) A Guideline of Selecting and Reporting Intraclass Correlation Coefficients for Reliability Research. J Chiropr Med 15:155-163

36. Bland JM, Altman DG (1999) Measuring agreement in method comparison studies. Stat Methods Med Res 8:135-160

37. Donal E, Lip GYH, Galderisi M et al (2016) EACVI/EHRA Expert Consensus Document on the role of multi-modality imaging for the evaluation of patients with atrial fibrillation. Eur Heart J Cardiovasc Imaging 17:355-383

38. Vincenti G, Monney P, Chaptinel J et al (2014) Compressed sensing single-breath-hold CMR for fast quantification of LV function, volumes, and mass. JACC Cardiovasc Imaging 7:882-892

39. Ma Y, Hou Y, Ma Q, Wang X, Sui S, Wang B (2019) Compressed SENSE single-breath-hold and free-breathing cine imaging for accelerated clinical evaluation of the left ventricle. Clin Radiol 74: 325.e9-325.e17

40. Ma LE, Markl M, Chow K et al (2019) Aortic 4D flow MRI in 2 minutes using compressed sensing, respiratory controlled adaptive k-space reordering, and inline reconstruction. Magn Reson Med 81:3675-3690

41. McElroy S, Ferrazzi G, Nazir MS et al (2020) Combined simultaneous multislice bSSFP and compressed sensing for first-pass myocardial perfusion at $1.5 \mathrm{~T}$ with high spatial resolution and coverage. Magn Reson Med. https://doi.org/10.1002/mrm.28345

42. Ferreira da Silva T, Galan-Arriola C, Montesinos P et al (2020) Single breath-hold saturation recovery 3D cardiac T1 mapping via compressed SENSE at 3 T. MAGMA. https://doi.org/10.1007/ s10334-020-00848-2

43. Tsao J, Boesiger P, Pruessmann KP (2003) k-t BLAST and k-t SENSE: dynamic MRI with high frame rate exploiting spatiotemporal correlations. Magn Reson Med 50:1031-1042

44. Huang F, Akao J, Vijayakumar S, Duensing GR, Limkeman M (2005) k-t GRAPPA: a k-space implementation for dynamic MRI with high reduction factor. Magn Reson Med 54:1172-1184

45. Feng L, Axel L, Chandarana H, Block KT, Sodickson DK, Otazo R (2016) XD-GRASP: golden-angle radial MRI with reconstruction of extra motion-state dimensions using compressed sensing. Magn Reson Med 75:775-788

46. Langton JEN, Lam H-I, Cowan BR et al (2017) Estimation of myocardial strain from non-rigid registration and highly accelerated cine CMR. Int J Cardiovasc Imaging 33:101-107

47. Fyrdahl A, Ramos JG, Eriksson MJ, Caidahl K Ugander M, Sigfridsson A (2020) Sector-wise golden-angle phase contrast with high temporal resolution for evaluation of left ventricular diastolic dysfunction. Magn Reson Med 83:1310-1321

48. Yang Y, Yin G, Jiang Y, Song L, Zhao S, Lu M (2020) Quantification of left atrial function in patients with nonobstructive hypertrophic cardiomyopathy by cardiovascular magnetic resonance feature tracking imaging: a feasibility and reproducibility study. J Cardiovasc Magn Reson 22:1-11 
49. Chirinos JA, Sardana M, Ansari B et al (2018) Left atrial phasic function by cardiac magnetic resonance feature tracking is a strong predictor of incident cardiovascular events. Circ Cardiovasc Imaging 11:e07512

50. Shang Y, Zhang X, Leng W et al (2018) Left atrium passive ejection fraction is the most sensitive index of type 2 diabetes mellitusrelated cardiac changes. Int J Cardiovasc Imaging 34:141-151

51. Pathan F, Zainal Abidin HA, Vo QH et al (2019) Left atrial strain: a multi-modality, multi-vendor comparison study. Eur Heart J Cardiovasc Imaging. https://doi.org/10.1093/ehjci/jez303
52. Sievers B, Kirchberg S, Addo M, Asli Bakan, Brandts B, Trappe H-J (2004) Assessment of left atrial volumes in sinus rhythm and atrial fibrillation using the biplane area-length method and cardiovascular magnetic resonance imaging with TrueFISP. J Cardiovasc Magn Reson 6:855-863

Publisher's note Springer Nature remains neutral with regard to jurisdictional claims in published maps and institutional affiliations. 